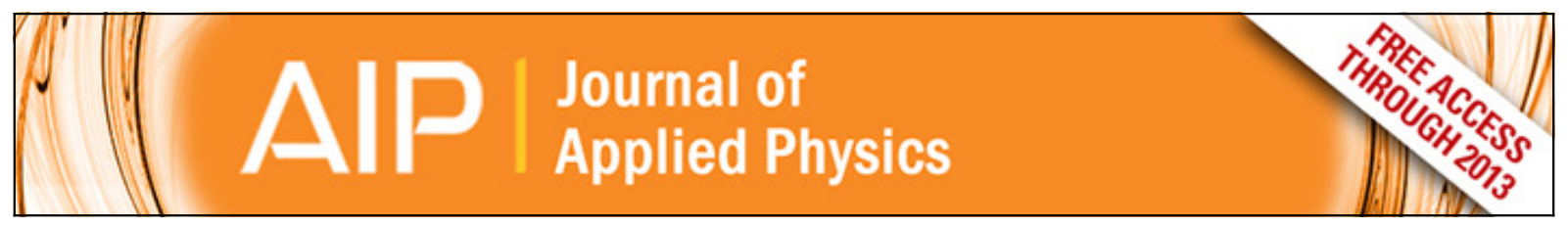

Strain and band edges in single and coupled cylindrical $\ln A s / G a A s$ and InP/InGaP selfassembled quantum dots

M. Tadi, F. M. Peeters, K. L. Janssens, M. Korkusiski, and P. Hawrylak

Citation: Journal of Applied Physics 92, 5819 (2002); doi: 10.1063/1.1510167

View online: http://dx.doi.org/10.1063/1.1510167

View Table of Contents: http://scitation.aip.org/content/aip/journal/jap/92/10?ver=pdfcov

Published by the AIP Publishing 


\title{
Strain and band edges in single and coupled cylindrical InAs/GaAs and InP/InGaP self-assembled quantum dots
}

\author{
M. Tadic, ${ }^{\text {a) }}$ F. M. Peeters, ${ }^{\text {b) }}$ and K. L. Janssens \\ Departement Natuurkunde, Universiteit Antwerpen (UIA), Universiteitsplein 1, B-2610 Antwerpen, Belgium \\ M. Korkusiński and P. Hawrylak \\ Institute for Microstructural Sciences, National Research Council, Ottawa, K1A 0R6, Canada
}

(Received 11 February 2002; accepted 30 July 2002)

\begin{abstract}
A comparative study is made of the strain distribution in cylindrical InAs/GaAs and $\mathrm{InP} / \mathrm{InGaP}$ self-assembled quantum dots as obtained from isotropic elasticity theory, the anisotropic continuum mechanical model, and from atomistic calculations. For the isotropic case, the recently proposed approach [J. H. Davies, J. Appl. Phys. 84, 1358 (1998)] is used, while the finite-element method, the valence force field method, and Stillinger-Weber potentials are employed to calculate the strain in anisotropic structures. We found that all four methods result in strain distributions of similar shapes, but with notable quantitative differences inside the dot and near the disk-matrix boundary. The variations of the diagonal strains with the height of the quantum dot, with fixed radius, as calculated from all models, are almost linear. Furthermore, the energies of the band edges in the two types of quantum dots are extracted from the multiband effective-mass theory by inserting the strain distributions as obtained by the four models. We demonstrated that all strain models produce effective potentials for the heavy and light holes which agree very well inside the dot. A negligible anisotropy of all normal strains in the $(x, y)$ plane is found, which, providing the axial symmetry of the kinetic part of the multiband effective-mass Hamiltonian, justifies the use of the axial approximation. Strain propagation along the vertical direction is also considered with the aim to study the influence of strain on the electron coupling in stacks of quantum dots. We found that the interaction between the strain fields of the individual quantum dots makes the effective quantum wells for the electrons in the conduction band shallower, thereby counteracting the quantum mechanical coupling. (C) 2002 American Institute of Physics. [DOI: 10.1063/1.1510167]
\end{abstract}

\section{INTRODUCTION}

The increased interest in self-assembled quantum dots (QD's) in recent years ${ }^{1,2}$ is driven by the prospective applications of these structures, e.g., lasers, ${ }^{3,4}$ infrared detectors, ${ }^{5}$ and resonant tunnelling devices. ${ }^{6}$ Due to the deltafunctionlike density of states, self-assembled QD's exhibit superior performance to quantum well structures. They are efficiently fabricated by the Stranski-Krastanow mode of epitaxial growth on lattice mismatched semiconductor substrates. In pseudomorphic quantum wells, the homogeneous strain is located only within the slab, while in self-assembled QD's, the strain fields generally have an inhomogeneous shape which spreads in both the dot and the semiconductor matrix in which the dot is incorporated. ${ }^{7}$ Furthermore, a single QD acts as a stressor for the growth of subsequent layers, thus promoting vertical ordering of QD's. ${ }^{8-12}$ The electron wavefunctions in the formed stacks are partially controlled by the strain, which is in turn affected by the number of dots in the vertical columns, their size, and the spacer thickness.

It has been experimentally determined that the StranskiKrastanow growth mode cannot be established for arbitrary

\footnotetext{
a) On leave from: Faculty of Electrical Engineering, University of Belgrade, Yugoslavia; electronic mail: tadic@uia.ua.ac.be

b)Electronic mail: peeters@uia.ua.ac.be
}

crystal orientation. ${ }^{13-16}$ For example, the growth of InAs on GaAs(110) and GaAs(111)A substrates is two-dimensional, leading to the formation of misfit dislocations when the thickness of the epitaxial film exceeds the critical value. ${ }^{13,14}$ On the contrary, three-dimensional (3D) strained InAs islands are formed during the growth on the (001) oriented GaAs substrates. ${ }^{15,16}$ In addition to InAs/GaAs QD's, ${ }^{8,11,15}$ InP/InGaP, ${ }^{12,17,18} \mathrm{GaAs} / \mathrm{GaSb},{ }^{19,20} \mathrm{Ge} / \mathrm{Si}^{21}{ }^{21}$ and $\mathrm{InAs} / \mathrm{InP}$ (Ref. 22) have all been demonstrated to produce selfassembled QD's. A free or cleaved surface may be in the vicinity of the capped $\mathrm{QD}$, which may have an impact on the strain distribution as recently analyzed. ${ }^{23-27}$ Those models prove that the interaction between the free surface and the QD plays an important role in the self-assembling process. ${ }^{23}$ By varying the growth conditions, self-assembled QD's of various shapes have been realized, but pyramidal and truncated pyramidal islands have been mainly considered theoretically, ${ }^{28-30}$ whereas less attention is given to the axially symmetric QD's, fabricated as lenses and disks. ${ }^{31,32}$ The conduction band in stacks of two cylindrical QD's produced by the flush-off technique has been theoretically considered very recently. ${ }^{33}$ Furthermore, the electronic structure in cylindrical InP/InGaP QD's has been calculated by the multiband effective-mass theory ${ }^{34}$ and those results were compared with recent magnetophotoluminescence measurements in a pulsed magnetic field. ${ }^{12}$ 
Prior to the calculation of the electronic structure in a self-assembled QD, the strain fields should be determined. A handful of various models which may sense the crystal anisotropy are at one's disposal. ${ }^{35-37}$ Analytical solutions exist only for a restricted set of QD shapes where the isotropic approximation is usually invoked. ${ }^{7,38}$ For other interesting isotropic structures, efficient numerical schemes are available, ${ }^{39,40}$ which, as recently demonstrated, may reproduce some details of the anisotropic strain calculations. ${ }^{40}$ For the latter, the anisotropic continuum mechanical model ${ }^{41}$ or the theory of inclusions ${ }^{35}$ might be employed. However, both these methods are not able to describe the strain at the level of the crystal unit cell, for which a more comprehensive atomistic approach is required. ${ }^{36,42}$

In the continuum mechanical (CM) model, the functional form of the elastic energy is minimized in order to obtain the distribution of the displacement in the structure, ${ }^{7,23,41}$ wherefrom the strain is extracted. Structures based not only on the zinc blende but also on the wurtzite semiconductors have been recently considered by this method. ${ }^{24}$ Without an extra cost, both finite difference and finite-element implementations of the CM model may handle the spatial dependence of the elastic constants in the structure. However, it was found that a CM-type calculation produces an artificial divergence of the strain at the apex of pyramidal QD's. ${ }^{29,41}$

In the atomistic theory, ${ }^{36,42-45}$ phenomenological expressions for the elastic energy which depends on the atomic coordinates and a set of empirical parameters are employed. ${ }^{29,41,43,46}$ The valence force field (VFF) model of Keating and Martin is the simplest approach of this kind, ${ }^{36,42}$ containing only two elastic parameters which are easily derived from the experimental elastic constants. The VFF model removes the divergence of the strain at the tip of the pyramid, and is able to recognize the true atomic symmetry which makes the distinction between the [110] and [1 10$]$ directions. $^{29,41}$ This model was recently used to extract the parameters of the isotropic elasticity relevant for the description of the epitaxial growth on thin compliant substrates. ${ }^{47,48}$

More complex approaches rely on Stillinger-Weber $(\mathrm{SW})^{43,49}$ or Tersoff potentials ${ }^{44,45,50}$ which have seven elastic parameters. Yu and Madhukar employed SW potentials in the molecular-dynamics simulation of $\mathrm{Ge}$ islands on $\mathrm{Si}^{49}$ They found that the contributions of the surface and volume terms to the total energy as calculated by their approach agree very well with the results of continuum theory, which was heuristically explained by Tersoff. ${ }^{51}$ A recent comparison between the VFF model and the SW models for the large pyramidal QD's showed that both models produce in-plane strains $\varepsilon_{x x}$ and $\varepsilon_{y y}$ which are of similar magnitude in the plane of the structure near the bottom of the pyramid, while the lower $\varepsilon_{z z}$ is computed by the SW calculation. ${ }^{46}$ However, along the vertical axis of the pyramid, the mutual relationship between the diagonal components of the strain tensor is altered, and the SW model leads to larger values for $\varepsilon_{x x}$ and $\varepsilon_{z z}$ in a large portion of the dot. ${ }^{46}$ The SW model, having a larger number of elastic parameters should a priori possess a higher accuracy than the VFF model. The main drawback of the atomistic models is the long computation time which is needed, limiting the size of the dot that can be analyzed.
Nevertheless, multimillion atomistic studies have been recently adopted to explain the experimental features of the vertical growth and lateral strain distribution in InAs QD's formed on GaAs mesas. ${ }^{52,53}$

The calculated strain serves as an input to the electronic structure calculation, for which various models may be employed. ${ }^{29,54-56}$ Among those, the effective-mass theory provides a clear explanation of the experimental results and can be implemented with relative ease. Multiband variants of this theory have been successfully adopted for selfassembled QD's. ${ }^{29,30}$ The electron and hole energies are extracted from the system of 3D partial differential equations, which represents the multiband effective-mass Hamiltonian. ${ }^{7,28-30}$ Nevertheless, a first account of the electron and hole confinement may be obtained from the strain induced edges of the conduction and valence bands. ${ }^{41,57}$

The aim of this article is to provide a detailed comparison of the strain distributions as obtained from the VFF, SW, and CM models for planar QD's and to compare their effect on the edges of the conduction and valence bands. A previous work was limited to InAs/GaAs and Ge/Si structures, whereas no attention has been devoted to a comparison of different strain models in type-II InP/InGaP QD's. In this article, we compare the strains in disk-shaped InAs/GaAs and InP/InGaP QD's calculated by the theory of isotropic elasticity (IE), ${ }^{40}$ anisotropic CM calculation, ${ }^{23,41}$ and the atomistic theory. ${ }^{29,36,41,42}$ The latter two models have been recently compared for pyramidal QD's, ${ }^{29,41}$ while the axial symmetry of QD's suggests that these two models should give comparable results to the isotropic continuum approach. ${ }^{40}$ The shapes, values, and the symmetries of different strain tensor components are determined and discussed. The strain fields in the elastically isotropic QD's are numerically determined by the method proposed in Ref. 40 while for the anisotropic case, the finite-element, ${ }^{58-61} \mathrm{VFF}$, and SW models are used. ${ }^{36,42}$ The elastic energy due to the bond deformation is described by the Keating expression in the VFF method. ${ }^{36}$ The investigated single QD structure of height $h$ and radius $R$ is schematically shown in Fig. 1(a). We performed our IE and CM calculations using cylindrical coordinates, but the strain tensor is expressed in Cartesian coordinates. The growth of the QD occurs along the [001] crystallographic direction, which is the $z$ axis in Fig. 1(a), while the $x$ and $y$ axes are oriented along the [100] and [010] directions, respectively. Such a choice of the coordinate system is consistent with both the elastic energy functional in the CM model and the multiband Hamiltonian employed to calculate the band edges.

In order to determine the spatial variation of the electron and hole band edges in the structure due to strain, we employed the effective-mass theory, taking into account the mixing between the heavy-hole and light-hole bands, while the electrons are considered decoupled from the hole bands. The influence of the in-plane anisotropy of the strain on the axial symmetry of the electron and hole band edges is explored. Such an analysis aims to consider the applicability of the axial approximation of the Pikus-Bir Hamiltonian, and also to assess the influence of the anisotropy of the strain on the band edges. The strain and band edges in the stacks con- 

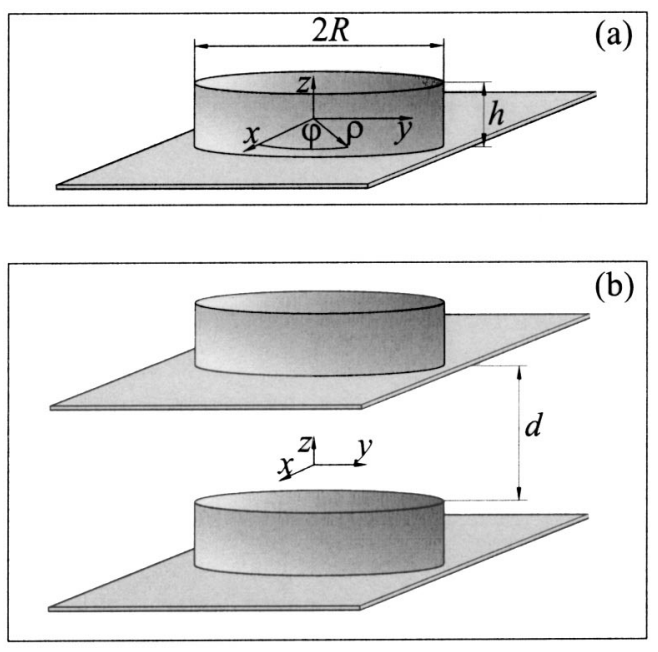

FIG. 1. (a) Schematic view of a single cylindrical QD of radius $R$ and height $h$. The wetting layer is depicted as the shaded plane which is discarded in our calculation. (b) A sketch of two vertically coupled QD's of radius $R$ and height $h$ separated by a spacer layer of thickness $d$.

sisting of the two vertically arranged QD's of equal size [see Fig. 1(b)] are also determined. We denote the separation between the two QD's as $d$ in Fig. 1(b). The wetting layers are depicted as the shaded planes in Figs. 1(a) and 1(b). Recent calculations indicated that due to their small thickness those layers can not substantially affect the distribution of the strain within the pyramidal QD's. ${ }^{23,30}$ Therefore, the wetting layers are discarded in our calculation, and the QD's are coherently embedded into the infinite matrix.

This article is organized as follows. In Sec. II, the continuum models to calculate the strain are described. The two atomistic approaches are explained in Sec. III. The procedure to calculate the band edges is explained in Sec. IV. The numerical results for the strain distributions and band edges calculated by the four models are compared and discussed in Sec. V.

\section{CONTINUUM MECHANICAL MODEL}

In order to compute the strain distribution in the structure shown in Fig. 1, we employ the following expression for the elastic energy of the large supercell of volume $V$ made up of cubic semiconductors: $:^{37,62}$

$$
\begin{aligned}
E_{\mathrm{el}}= & \int_{V} d V\left[\frac{1}{2} C_{11}\left(\varepsilon_{x x}^{2}+\varepsilon_{y y}^{2}+\varepsilon_{z z}^{2}\right)+C_{12}\left(\varepsilon_{x x} \varepsilon_{y y}+\varepsilon_{y y} \varepsilon_{z z}\right.\right. \\
& \left.+\varepsilon_{z z} \varepsilon_{x x}\right)+2 C_{44}\left(\varepsilon_{x y}^{2}+\varepsilon_{y z}^{2}+\varepsilon_{z x}^{2}\right)-2 \alpha\left(\varepsilon_{x x}+\varepsilon_{y y}\right. \\
& \left.\left.+\varepsilon_{z z}\right) \varepsilon_{0}+3 \alpha \varepsilon_{0}^{2}\right] .
\end{aligned}
$$

Here $\varepsilon_{i j}$ denotes the $i j$ component of the strain tensor ( $i$ $=x, y, z), C_{i j}$ is the elastic modulus tensor in the reduced notation, $\alpha=C_{11} / 2+C_{12}$ in the dot and zero in the matrix, and $\varepsilon_{0}$ denotes the misfit strain between the dot and the barrier. According to the procedure to derive Eq. (1), ${ }^{40,62} \varepsilon_{0}$ is defined as:

$$
\varepsilon_{0}=\left(a_{d}-a_{m}\right) / a_{m},
$$

where $a_{d}$ and $a_{m}$ denote the lattice constant in the dot and the matrix, respectively.

The components $\varepsilon_{i j}$ are related to the displacement vector $\mathbf{u}=\left(u_{x}, u_{y}, u_{z}\right)$ as:

$$
\varepsilon_{i j}=\frac{1}{2}\left(\frac{\partial u_{i}}{\partial r_{j}}+\frac{\partial u_{j}}{\partial r_{i}}\right)
$$

where $r_{i}$ denotes the $x, y$, or $z$ component of the radius vector. In addition to the nine terms in the strain tensor, we define the lateral strain (half of the in-plane strain defined in Ref. 63) as:

$$
\varepsilon_{\|}=\frac{1}{2}\left(\varepsilon_{x x}+\varepsilon_{y y}\right)
$$

The minimization of the elastic energy given by Eq. (1), is manageable only if it is supplemented by boundary conditions. Regular periodicity of the supercell in the plane of the layer or in all three directions is usually assumed for this purpose. ${ }^{41}$ Providing a sufficiently large supercell, however, the rigid boundary conditions may equally well be applied. ${ }^{61}$ This approach may require more discretization points but avoids potentially superfluous interaction between the strain fields of the individual QD's in an artificial QD array.

The minimization of the elastic energy generates a system of linear equations:

$$
\frac{d E_{\mathrm{el}}}{d u_{x(i j k)}}=\frac{d E_{\mathrm{el}}}{d u_{y(i j k)}}=\frac{d E_{\mathrm{el}}}{d u_{z(i j k)}}=0,
$$

where $u_{\eta(i j k)}$ denotes the component of the displacement vector along the $\eta$ direction at the ijk vertex. A uniform mesh is applied in the $\varphi$ direction, while in both the $\rho$ and the $z$ directions, a nonuniform mesh, with increased density near the dot boundary is adopted. Due to the symmetry of the structure, the calculation is performed in the first octant only, with the normal components of the displacement vector equal to zero at the symmetry planes. Furthermore, rigid boundary conditions are applied at the surface of the discretization cylinder, i.e., the displacement vector is taken to be equal to zero at $\rho=R_{t}$ and $z=L_{z}$. The mesh comprises 33 vertices in the $\varphi$ direction, and 65 in both the $\rho$ and $z$ directions, which leads to a system of about $4 \times 10^{5}$ linear equations. The recent $\mathrm{CM}$ calculations for truncated pyramidal QD's employed $10^{4}$ elements, ${ }^{60,61}$ while we generated about $1.4 \times 10^{5}$ elements in our calculation, which is sufficient to overcome the lack of the mesh adaptation. Furthermore, the method is tested by comparing with the IE theory, ${ }^{40}$ and, as demonstrated next, a good agreement between the two is found. This system is efficiently solved by the preconditioned conjugate gradient method, and the strains are computed at the centers of the elements.

The strain distribution in an isotropic crystal may, in principle, be found by minimizing $E_{\mathrm{el}}$ as given by Eq. (1). But we want to test the method proposed in Ref. 40, which considers the strain in an arbitrary shaped elastically isotropic QD by using the analogy with electrostatics. The integral expressions for the displacement and the strain are derived from the analytical strain distribution around a spherical inclusion and by using the superposition principle. ${ }^{40}$ Supported 
TABLE I. Experimental values of the material parameters for the InAs/GaAs and InP/InGaP QD's. In InAs/ GaAs QD's, the values given in Ref. 29 are used. For InP/InGaP QD's, all parameters have the same values as in Ref. 30. Elastic parameters of the two atomistic calculations are also given. Those for the VFF model are calculated as in Ref. 2, while the parameters of the SW potential are taken from Ref. 65. The results for the InGaP alloy are obtained by averaging between the values for InP and $\mathrm{GaP}$ as given by Eqs. (8a)-(8e).

\begin{tabular}{ccccc}
\hline \hline Parameter & InAs & GaAs & InP & $\mathrm{In}_{0.49} \mathrm{Ga}_{0.51} \mathrm{P}$ \\
\hline$a_{0}(\mathrm{~nm})$ & 0.60584 & 0.56532 & 0.58687 & 0.56532 \\
$E_{g}(\mathrm{eV})$ & 0.413 & 1.518 & 1.424 & 1.97 \\
$a_{c}(\mathrm{eV})$ & -6.66 & -9.3 & -7.0 & -7.5 \\
$a_{v}(\mathrm{eV})$ & 0.66 & 0.7 & 0.4 & 0.4 \\
$b(\mathrm{eV})$ & -1.8 & -2.0 & -2.0 & -1.9 \\
$d(\mathrm{eV})$ & -3.6 & -5.4 & -5.0 & -4.75 \\
$C_{11}\left(10^{10} \mathrm{~N} / \mathrm{m}^{2}\right)$ & 8.329 & 12.11 & 10.22 & 12.17 \\
$C_{12}\left(10^{10} \mathrm{~N} / \mathrm{m}^{2}\right)$ & 4.526 & 5.48 & 5.76 & 6.01 \\
$C_{44}\left(10^{10} \mathrm{~N} / \mathrm{m}^{2}\right)$ & 3.959 & 6.04 & 4.6 & 5.82 \\
$\alpha(\mathrm{N} / \mathrm{m})$ & 33.18 & 40.35 & 40.35 & 42.68 \\
$\beta(\mathrm{N} / \mathrm{m})$ & 5.76 & 9.37 & 6.54 & 8.71 \\
$\varepsilon(\mathrm{nm})$ & 1.55 & 1.63 & 1.74 & 1.76 \\
$A$ & 7.80995 & 7.73502 & 7.90793 & 7.76148 \\
$B$ & 0.706 & 0.696 & 0.719 & 0.699 \\
$\sigma(\mathrm{nm})$ & 0.22819 & 0.21342 & 0.22046 & 0.21330 \\
$\lambda(\mathrm{nm})$ & 22.85 & 30.25 & 22.11 & 25.64 \\
\hline \hline
\end{tabular}

by the experimental data displayed in Table I, the Poisson ratio $\nu$ approximately equals one third for all employed semiconductors. By adopting $\nu=1 / 3$, the elastic constants in the isotropic semiconductors are related by:

$$
C_{12}=\frac{C_{11}}{2}, \quad C_{44}=\frac{C_{11}}{4} .
$$

In order to test our CM calculation, $C_{12}$ and $C_{44}$ are computed for the given $C_{11}$ in the matrix and those elastic constants are also used for the disk.

All components of the Cartesian strain tensor in QD's vary with the in-plane angle $\varphi$, but in cylindrical coordinates, the component of the displacement vector along the azimuthal angle in elastically isotropic crystals is equal to zero, $u_{\varphi}=0$. Therefore, the lateral strain in the isotropic structure is given by:

$$
\varepsilon_{\|}=\frac{1}{2}\left(\varepsilon_{x x}+\varepsilon_{y y}\right)=\frac{1}{2}\left(\varepsilon_{\rho \rho}+\varepsilon_{\varphi \varphi}\right)=\frac{1}{2 \rho} \frac{\partial}{\partial \rho}\left(\rho u_{\rho}\right) .
$$

Since neither $\varepsilon_{\|}$nor $\varepsilon_{z z}$ depend on the azimuthal angle, the IE calculation of these strains is reduced to two dimensions. We actually calculated only these two strains in our IE approach, for which we used the logarithmically weighted finite difference technique which we developed earlier. ${ }^{64}$

\section{ATOMISTIC MODELS}

In our VFF and SW simulations, about 3.6 million atoms located in a supercell with $40 \times 40 \mathrm{~nm}^{2}$ base and height of 52 $\mathrm{nm}$ were considered. The elastic energy was relaxed by simultaneously moving all the atoms, except the atoms at the boundary of the supercell. The line minimization in the conjugate gradient method was employed as described in Ref. 41. The form of the SW potential is exponential, and the numerical line search of the energy minimum is necessary, which increases the computational time dramatically for such a large number of atoms. Therefore, we limited ourselves to the calculation of only 7 monolayer (ML) high InAs/GaAs and InP/InGaP QD's. When the distribution of the atoms is determined, the strain is computed on the mesh formed by the cation sites by calculating the bond vectors between the four anion atoms surrounding the cation atom as proposed in Ref. 41.

Two parameters of the Keating's potential in the VFF method, $\alpha$ and $\beta,{ }^{36}$ cannot be uniquely related to the three experimental elastic constants. These parameters are usually extracted from the experimental values of $C_{11}$ and $C_{12}$ in each material. ${ }^{2}$ For the bonds crossing the boundary, the parameters of the interatomic potential are determined as the geometrical averages of the values in the dot and the matrix. We also performed the calculation of the strain distribution by the CM model with the experimental values of $C_{11}$ and $C_{12}$, and $C_{44}$ extracted from the elastic parameters of the Keating's potential. ${ }^{2}$ We denote these calculations as CV.

The SW potential contains seven empirical parameters, $A, B, \sigma, \lambda, \gamma, a$, and $\varepsilon .{ }^{43}$ These parameters for InP, GaP, InAs, and GaAs self-assembled QD's were determined in Ref. 65. Since the data for $\operatorname{In}_{0.49} \mathrm{Ga}_{0.51} \mathrm{P}$ are not available, we employed the averaging procedure of the data for the two constituent binaries in the alloy:

$$
\begin{aligned}
& A_{\mathrm{InGaP}}=A_{\mathrm{InP}}^{0.49} A_{\mathrm{GaP}}^{0.51}, \\
& B_{\mathrm{InGaP}}=B_{\mathrm{InP}}^{0.49} B_{\mathrm{GaP}}^{0.51}, \\
& \varepsilon_{\mathrm{InGaP}}=\varepsilon_{\mathrm{InP}}^{0.49} \varepsilon_{\mathrm{GaP}}^{0.51}, \\
& \lambda_{\mathrm{InGaP}}=\lambda_{\mathrm{InP}}^{0.49} \lambda_{\mathrm{GaP}}^{0.51}, \\
& \sigma_{\mathrm{InGaP}}=0.49 \times \sigma_{\mathrm{InP}}+0.51 \times \sigma_{\mathrm{GaP}} .
\end{aligned}
$$

Such calculation of the elastic parameters of InGaP is consistent with the calculation of the interatomic potential at the dot boundary proposed in Ref. 66 for Ge/Si structures. The 
parameters for the four employed semiconductors are given in Table I. The same values of parameters $a$ and $\gamma$ are used for all four semiconductors, $a=1.8$ and $\gamma=1.2$, as originally proposed by Stillinger and Weber ${ }^{43}$ and used by Ichimura. ${ }^{65}$

\section{BAND EDGES}

We assume that the conduction band is decoupled from the valence bands, but take into account the mixing between the bands of the heavy and light holes. The edge of the conduction band responds only to the hydrostatic strain

$$
E_{c}(x, y, z)=E_{c 0}(x, y, z)+a_{c} \varepsilon_{\text {hyd }}(x, y, z),
$$

where $E_{c 0}$ is a variation of the band edge due to the band offset, $a_{c}$ denotes the hydrostatic deformation potential for the conduction band, and $\varepsilon_{\text {hyd }}$ is the hydrostatic strain $\left(\varepsilon_{\text {hyd }}=\varepsilon_{x x}+\varepsilon_{y y}+\varepsilon_{z z}\right)$. The heavy- and light-hole band edges are extracted from the Pikus-Bir Hamiltonian ${ }^{67,68}$

$$
H=\left[\begin{array}{cccc}
P+Q & S & 0 & -R \\
S^{\dagger} & P-Q & R & 0 \\
0 & R^{\dagger} & P+Q & S^{\dagger} \\
-R^{\dagger} & 0 & S & P-Q
\end{array}\right] \begin{gathered}
|3 / 2,3 / 2\rangle \\
|3 / 2,1 / 2\rangle \\
|3 / 2,-3 / 2\rangle, \\
|3 / 2,-1 / 2\rangle
\end{gathered}
$$

where the matrix elements are calculated for the zero-hole wavevector $k=0$. The periodic parts of the Bloch functions are written explicitly at the right-hand side of Eq. (10). The matrix elements of the Hamiltonian (10) are given by ${ }^{67}$

$$
\begin{aligned}
& P=a_{v}\left(\varepsilon_{x x}+\varepsilon_{y y}+\varepsilon_{z z}\right), \\
& Q=\frac{b}{2}\left(\varepsilon_{x x}+\varepsilon_{y y}-2 \varepsilon_{z z}\right), \\
& R=-\frac{\sqrt{3}}{2} b\left(\varepsilon_{x x}-\varepsilon_{y y}\right)+i d \varepsilon_{x y}, \\
& S=-d\left(\varepsilon_{z x}-i \varepsilon_{y z}\right) .
\end{aligned}
$$

Here $a_{v}, b$, and $d$ denote the valence band deformation potentials. The band edges of the valence bands are given by

$$
\begin{aligned}
& E_{v \mathrm{hh}}=E_{v 0}(x, y, z)+P+\operatorname{sgn}(Q) \sqrt{Q^{2}+R R^{\dagger}+S S^{\dagger}}, \\
& E_{v \mathrm{lh}}=E_{v 0}(x, y, z)+P-\operatorname{sgn}(Q) \sqrt{Q^{2}+R R^{\dagger}+S S^{\dagger}},
\end{aligned}
$$

where $\mathrm{hh}$ and $\mathrm{lh}$ denote the heavy and light holes, respectively, and $E_{v 0}$ is the part due to the valence band offset between the dot and the matrix semiconductor.

Provided the full axial symmetry of the kinetic Hamiltonian, the electronic structure calculation may be alleviated by excluding the part which depends on the angular coordinate. ${ }^{34}$ The homogeneous biaxial strain in pseudomorphic slabs does not break the axial symmetry, and the axial approximation is successfully applied there. ${ }^{69,70}$ Whether the axial approximation is applicable to the inhomogeneous strain distribution in QD's, can be qualitatively inferred from the band edges.

\section{NUMERICAL RESULTS AND DISCUSSION}

The four models (IE, CM, VFF, and SW) supplemented with the $\mathrm{CV}$ calculation are tested for the InAs/GaAs and
InP/InGaP QD's grown on $\{001\}$ substrates. The experimental elastic and lattice constants of the four semiconductors which make up these structures are given in Table I. The $\alpha$ and $\beta$ are computed as in Ref. 2, while the parameters of the SW potentials for the four binaries are taken from Ref. 65 and for the $\mathrm{InGaP}$ are computed using Eqs. (8a)-(8e). The strain calculated by the CM model is invariant to the QD's scaling, and the deviation of the VFF model from this rule is expected to be rather small. ${ }^{71}$ Therefore, we explored the strain fields as they vary with the QD height in the range 2-13 ML's with a step size equal to a single atomic monolayer, while the QD radius is kept fixed at $R=8 \mathrm{~nm}$. A compelling reason for the application of such a procedure is higher sensitivity of the electron and hole levels to the change of the QD height than to the change of its radius. In the $\mathrm{CM}$ model, the QD radius and the height are continuous variables, implying that the atomic interface between the two layers may be considered abrupt. In reality, the interface is shared between the atoms of two species, and for a closer comparison with the VFF model, the CM model usually employs a mesh commensurate with the atomic positions. ${ }^{29,41}$

In order to fully use the capability of the CM model to work with a nonuniform mesh, we assume that the sides and bases of the cylinder are perfectly defined in the IE, CM, and $\mathrm{CV}$ calculations. The atomic arrangement does not comply to the perfect axial symmetry. This deviation which arises from the different symmetries of the structure and the crystal unit cell are unimportant for the conclusions derived in this section. As a matter of fact, the radius of the QD is larger than the height for the whole explored range of QD heights, and the assumption of the axial symmetry in the three continuum models can not substantially alter the strain field. If not explicitly indicated, all figures presented next address the case of the 7 ML high QD. Moreover, due to a limited space in the figures, we employed shorthand notations IG and II to refer to the InAs/GaAs and InP/InGaP QD, respectively.

In order to test our CM calculation, we used the values for the elastic constants of the elastically isotropic structures, as explained in Sec. II, and we compared the results of this calculation with the IE theory. The deviation of the CM result from the axial symmetry is shown by plotting the angular averages of $\varepsilon_{\|}$and $\varepsilon_{z z}$ :

$$
\left\langle\varepsilon_{\|}\right\rangle=\frac{1}{2 \pi} \int_{0}^{2 \pi} \varepsilon_{\|} d \varphi, \quad\left\langle\varepsilon_{z z}\right\rangle=\frac{1}{2 \pi} \int_{0}^{2 \pi} \varepsilon_{z z} d \varphi,
$$

in Figs. 2(a) and 2(b). Very good agreement between the two models is found along the whole $\rho$ axis, while the small discrepancy near the boundary is due to the small deviation of the CM calculated strains from axial symmetry, the different step size used in the IE and CM models, and the finite number of mesh vertices used to average the strains displayed in Fig. 2 over the $\varphi$ direction.

In order to provide the background for the subsequent analyses of the band edges, the distribution of the strain in the $z=0$ plane is examined by the CM model. The nonzero terms of the strain tensor in the InP/InGaP QD are shown in Fig. 3. The normal strains $\varepsilon_{x x}, \varepsilon_{y y}$, and $\varepsilon_{z z}$ are shown in Figs. 3(a), 3(b), and 3(c), respectively. In addition, the lateral strain is given in Fig. 3(d). One may notice that both $\varepsilon_{x x}$ and 


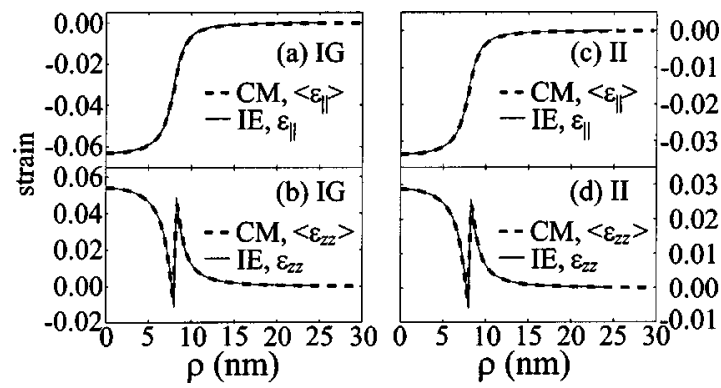

FIG. 2. Comparison between the variation of the strains in the $\mathrm{z}=0$ plane calculated from the CM model (thick dashed curves) and the IE theory (thin solid curves). For the CM calculation, the strain averaged over the angular coordinate is shown. Left-hand side panel: Results for the InAs/GaAs QD. (a) The variation of the $\varepsilon_{\|}$and $\left\langle\varepsilon_{\|}\right\rangle$along the $\rho$ direction. (b) The distributions of $\varepsilon_{z z}$ and $\left\langle\varepsilon_{z z}\right\rangle$. Right-hand side panel: The strains in the InP/InGaP QD. (c) The calculated variations of $\varepsilon_{\|}$and $\left\langle\varepsilon_{\|}\right\rangle$. (d) $\varepsilon_{z z}$ and $\left\langle\varepsilon_{z z}\right\rangle$ extracted from the two models.

$\varepsilon_{y y}$ lack in-plane isotropy, but both $\varepsilon_{\|}$and $\varepsilon_{z z}$ are almost axially symmetric in the $\mathrm{z}=0$ plane. Due to the symmetry of the disk, only $\varepsilon_{x y}$ is finite, while the other off-diagonal components of the strain tensor are zero in the $\mathrm{z}=0$ plane. Unlike the normal strains, $\varepsilon_{x y}$ is small inside the dot and in the matrix, as demonstrated in Fig. 3(e). Tensile shear strain exists in the disk, while it is compressive in the matrix. The profile of the shear strain $\varepsilon_{x y}$ is smooth in both the disk and the matrix, but its sign alters sharply at the radial boundary
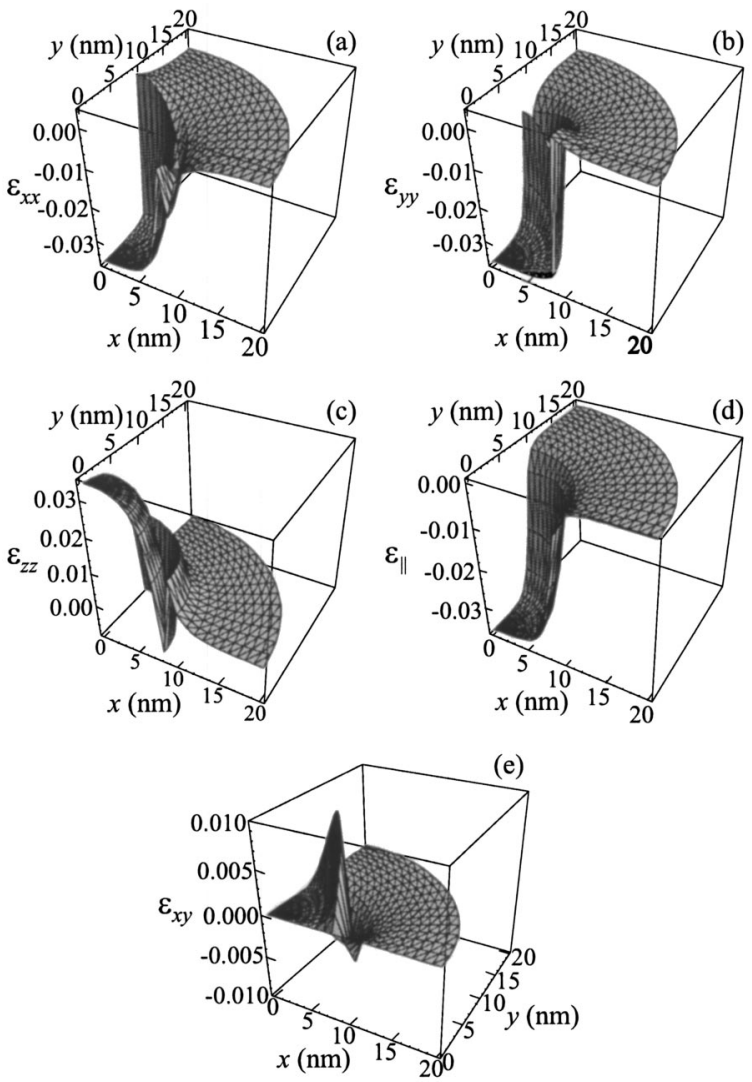

FIG. 3. Plots of the CM calculated strain tensor components in a $7 \mathrm{ML}$ high $\mathrm{InP} / \mathrm{InGaP} \mathrm{QD}$ of radius $R=8 \mathrm{~nm}$ in the $z=0$ plane: (a) $\varepsilon_{x x}$, (b) $\varepsilon_{y y}$, (c) $\varepsilon_{z z}$, (d) $\varepsilon_{\|}=\left(\varepsilon_{x x}+\varepsilon_{y y}\right) / 2$, and (e) $\varepsilon_{x y}$.

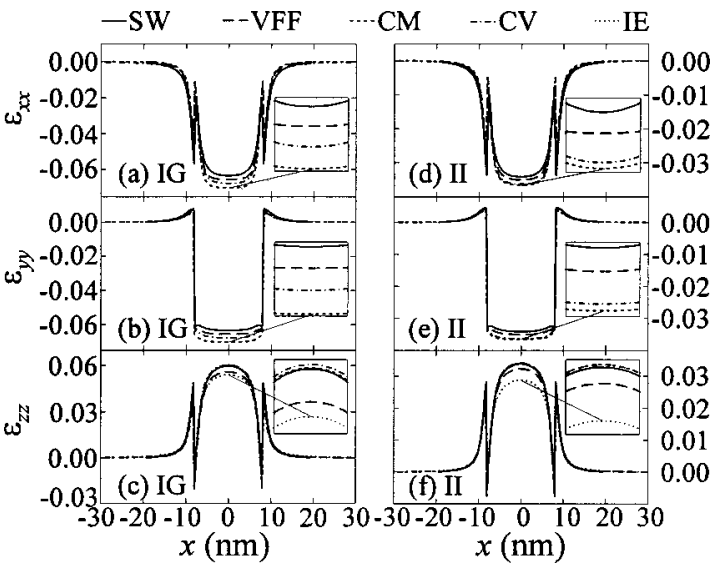

FIG. 4. $\varepsilon_{x x}$ and $\varepsilon_{y y}$ obtained by the CM, CV, SW, and VFF models along the [100] direction, and comparison between the IE theory, CM, CV, SW, and VFF models for the calculation of $\varepsilon_{z z}$. Left-hand side panel: The normal components of the strain tensor in InAs/GaAs: (a) $\varepsilon_{x x}$, (b) $\varepsilon_{y y}$, and (c) $\varepsilon_{z z}$. Right-hand side panel: The normal strains in the InP/InGaP QD. (d) $\varepsilon_{x x}$, (e) $\varepsilon_{y y}$, and (f) $\varepsilon_{z z}$. The solid lines in all figures represent the SW result, the long dashed curves are obtained by the VFF method, the short dashed curves denote the CM results, the dotted-dashed curves denote the results of the $\mathrm{CV}$ calculation, while the results for the elastically isotropic crystals are denoted by the dotted lines. The height of the QD was 7 MLs and its radius equals $8 \mathrm{~nm}$. Due to the symmetry, all shear strains are equal to zero.

of the disk, where it reaches its maximum and minimum values in the $\varphi=\pi / 4$ direction.

The normal strains calculated by the five models along the [100] direction in the InAs/GaAs QD are shown in the left-hand side panel of Fig. 4 [Figs. 4(a)-4(c)], while Figs. 4(d)-4(f) show the strain distributions in the InP/InGaP QD's. Due to symmetry, all shear strains are equal to zero. As already explained, our IE computation gives only $\varepsilon_{\|}$and $\varepsilon_{z z}$, therefore, only the other four calculations are compared in Figs. 4(a), 4(b), 4(d), and 4(e), while the results of all five calculations are given in Figs. 4(c) and 4(f). The $\varepsilon_{x x}$ strain of the smallest magnitude is obtained from the SW model. The ordering of the distributions of $\varepsilon_{y y}$ along the [100] direction calculated by the four methods is the same as the ordering found for $\varepsilon_{x x}$. However, there is a difference in the shape between these two strain tensor components. $\varepsilon_{x x}$ is compressive along the whole $x$ axis, whereas $\varepsilon_{y y}$ is compressive inside the QD, but tensile in the barrier. The $\varepsilon_{z z}$ exhibits almost an inverted behavior as compared to $\varepsilon_{x x}$. Our results for $\varepsilon_{z z}$ show that the largest $\varepsilon_{z z}$ is produced by the CM and $\mathrm{CV}$ models which almost coincide for the used value of the QD height, and also coincides with the distribution of $\varepsilon_{z z}$ calculated by the comprehensive SW model. $\varepsilon_{z z}$ computed by the VFF model is lower than SW, which contradicts what was found for the large pyramidal QD's. The difference between our results and those published in Ref. 46 is understandable since our structure is planar. But the relative magnitudes of the different strains within the dot correspond to the $z$ direction of the pyramidal QD's, and the sign of this ratio minus unity is the same for both the [001] and [100] directions. The lowest $\varepsilon_{z z}$ inside the dot is computed from the isotropic model. All five models, however, exhibit rather good mutual agreement in the matrix. 


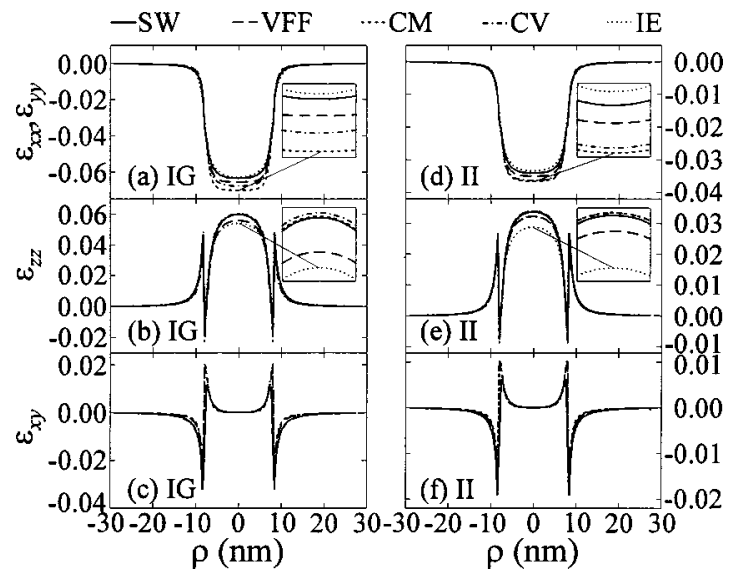

FIG. 5. Strains obtained by the five models along the [110] direction in a 7 ML high QD, with $8 \mathrm{~nm}$ radius. For the $\varepsilon_{x y}$ only the results of the SW, VFF, $\mathrm{CV}$, and CM models are shown. Due to the symmetry, the curves for $\varepsilon_{y y}$ and $\varepsilon_{x x}$ coincide. Left-hand side panel: Strains in the InAs/GaAs QD: (a) $\varepsilon_{x x}$ and $\varepsilon_{y y}$, (b) $\varepsilon_{z z}$, and (c) $\varepsilon_{x y}$. Right-hand side panel: Strains in the InP/ InGaP QD: (d) $\varepsilon_{x x}$ and $\varepsilon_{y y}$, (e) $\varepsilon_{z z}$, and (f) $\varepsilon_{x y}$. Other shear strains are equal to zero along this direction. The same notations as in Fig. 4 are used.

In order to inspect the anisotropy and to compare the four models in more detail, in Figs. 5(a)-5(f) we give the variation of $\varepsilon_{x x}, \varepsilon_{z z}$, and $\varepsilon_{x y}$ along the [110] direction. Due to the symmetry, we have $\varepsilon_{x x} \equiv \varepsilon_{y y}, \varepsilon_{y z}=0$, and $\varepsilon_{z x}=0$. For the $\varepsilon_{x y}$ shown in Figs. 5(c) and 5(f) only the results of the $\mathrm{SW}$, VFF, CM, and CV calculations are compared. The strains in the $7 \mathrm{ML}$ high InAs/GaAs and InP/InGaP QD's are shown in Figs. 5(a)-5(c) and 5(d)-5(f), respectively. The profile of $\varepsilon_{x x}$ in this direction is smooth [compare Figs. 5(a) and 4(a)], while $\varepsilon_{z z}$ and $\varepsilon_{x y}$ vary abruptly across the lateral side of the QD. Quite interestingly, the agreement between the SW and a simple IE calculation is rather good. The curves in Figs. 5(c) and 5(f) obtained by the two atomistic calculations are very close to each other, and they almost coincide with the curves produced by the CV model.

Similar to the strains along the [100] and [110] directions, the four models deliver qualitatively similar strains along the [001] direction. This is demonstrated in Figs. 6(a) and 6(b), where the strain distributions in the InAs/GaAs QD are shown, and in Figs. 6(c) and 6(d) for the InP/InGaP single QD. Due to the symmetry, all shear strains are zero, and therefore, also the IE result is shown. Distributions of $\varepsilon_{x x}$ obtained by the SW and VFF models are very close to each other, and also the two distributions obtained by the CM and $\mathrm{CV}$ models. The relative values of the strains for both $\varepsilon_{x x}$ and $\varepsilon_{z z}$ inside the dot calculated by the VFF and SW models agree with the strain distributions along the $z$ direction obtained in Ref. 46. The peak in $\varepsilon_{x x}$ is produced in the matrix by all models except in the IE theory. According to both the VFF and SW models, the sign of $\varepsilon_{z z}$ does not sharply alter at the boundary, but decays into the matrix in a nonabrupt fashion, while according to the $\mathrm{CM}, \mathrm{CV}$, and IE models a perfect discontinuity of $\varepsilon_{z z}$ exists at the base of the disk.

The edge of the conduction band in the InAs/GaAs QD along the [100] and [110] directions is shown in Figs. 7(a) and 7(b), respectively. Figures 7(c) and 7(d) display the con-

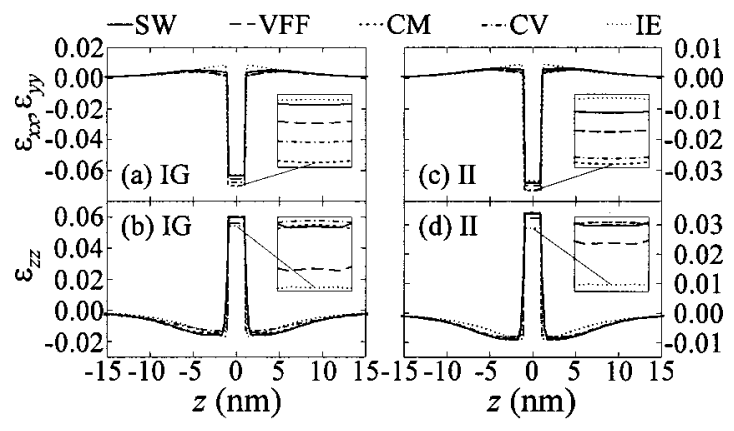

FIG. 6. The normal strains along the [001] direction. Due to the symmetry, $\varepsilon_{y y}=\varepsilon_{x x}$. Left-hand side panel: The normal strains in the InAs/GaAs QD: (a) $\varepsilon_{x x}$ and $\varepsilon_{y y}$ and (b) $\varepsilon_{z z}$. Right-hand side panel: Three components of the strain tensor in the InP/InGaP QD: (c) $\varepsilon_{x x}$ and $\varepsilon_{y y}$, (d) $\varepsilon_{z z}$. All shear strain components are equal to zero along the $z$ axis. The same notations as in Fig. 4 are employed in this figure.

duction band edge in the InP/InGaP QD for the two chosen directions. Our results from the IE theory may be used in the axial approximation of the multiband effective-mass model ${ }^{34}$ and the effective potentials calculated for the axially symmetric Hamiltonian with the strain determined by the IE theory are shown in Figs. 7(a)-7(d). The effective potential well in the conduction band is shallowest if the CM model is employed for the calculation, and the agreement between the $\mathrm{CM}, \mathrm{CV}$, and the VFF calculation is better for the $\mathrm{InP} / \mathrm{InGaP}$ QD. Furthermore, almost the same shapes of the strain distributions are found for both directions shown in Figs. 7(a)7(d) demonstrating that the conduction band edge is almost independent of the direction in the $z=0$ plane, except near the disk boundary. Furthermore, smooth profiles are produced along the [110] directions by all methods and are shown in Figs. 7(b) and 7(d). This indicates that the oscillations in $\varepsilon_{z z}$ and $\varepsilon_{x y}$, which were shown in Fig. 5, cancel each other out in Eqs. (12a) and (12b).

The valence band edges computed from the five models are shown in Figs. 8(a) and 8(b) for the InAs/GaAs QD, and in Figs. 8(c) and 8(d) for the InP/InGaP QD. As in Fig. 7, the effective potentials for the heavy and the light holes are shown for the strain distribution determined by the IE theory. Because of the different lattice mismatch and different va-

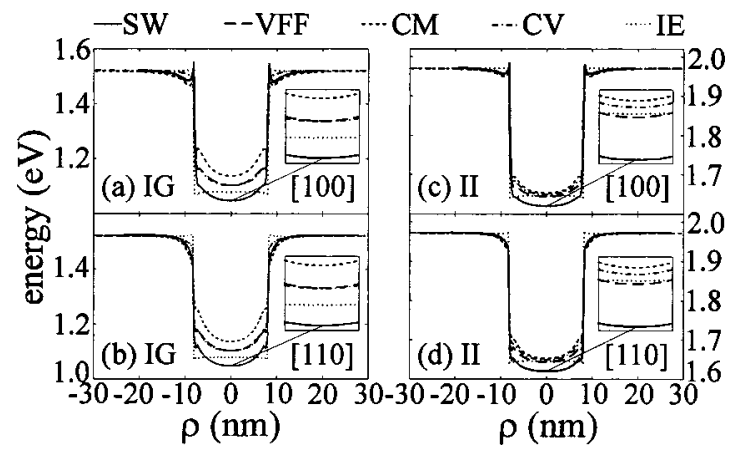

FIG. 7. The edge of the conduction band along the [100] and [110] directions in the InAs/GaAs and the InP/InGaP QD. Left-hand side panel: The edge of the conduction band in InAs/GaAs QD along: (a) the [100] and (b) the [110] direction. Right-hand side panel: The edge of the conduction band in the InP/InGaP QD along: (c) the [100] and (d) the [110] direction. The same notations as in Fig. 4 are employed. 


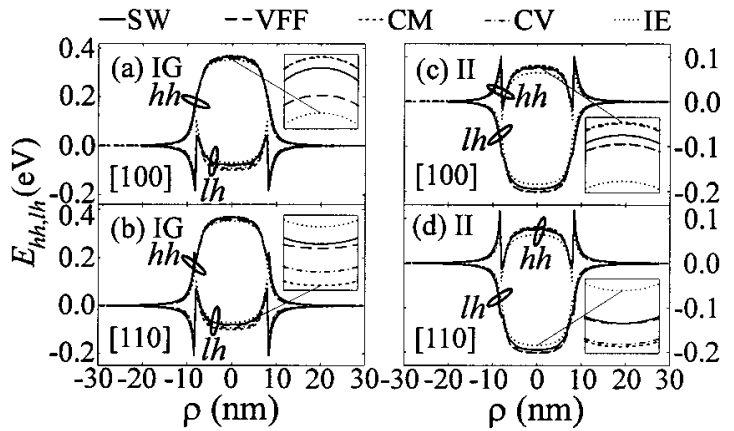

FIG. 8. The heavy- and light-hole band edges in InAs/GaAs (left-hand side panel) and $\mathrm{InP} / \mathrm{InGaP}$ (right-hand side panel) QD's calculated by the five models along the [100] and [110] directions. Left-hand side panel: The heavy-hole and light-hole band edges in the InAs/GaAs QD along: (a) the [100] and (b) the [110] direction. Right-hand side panel: Band edges of the heavy- and light-hole bands in InP/InGaP QD's along: (c) the [100] direction, and (d) the [110] direction. The same notations as in Fig. 4 are employed.

lence band offsets in the two QD's, the shapes of the curves in the left-hand side panel do not match those in the righthand side panel, which is essentially different from the conduction band case [Figs. 7(a)-7(d)]. For both investigated QD's, the strain brings about the localization of the heavy holes inside the QD, but the difference between the two QD's occurs near the boundary, where the InP/InGaP QD exhibits two satellite quantum wells for the heavy holes in addition to the central one, while such potential wells are almost missing or very narrow in InAs/GaAs. All models indicate that the light holes in the InAs/GaAs QD's are localized near the lateral side of the disk, whereas the effective potential does not confine the light holes in the $z=0$ plane of the InP/InGaP QD.

The conduction band edge as it varies along the $z$ direction is shown in Figs. 9(a) and 9(b). Due to the absence of the shear strains along this direction, the effective potentials for both the electrons and holes coincide with the conduction and the valence band edges. Surprisingly, the conduction band edge in the center of the QD calculated by the IE theory is positioned between the VFF and SW result for InAs/GaAs QD's and is slightly above the VFF produced conduction band edge for InP/InGaP QD's. Due to the nonabrupt variation of the strain tensor components delivered by the two atomistic approaches shown in Fig. 6, and the slower decrease of $\varepsilon_{z z}$ calculated by the atomistic approaches, SW and VFF profiles are nonabrupt at the disk boundary but have a

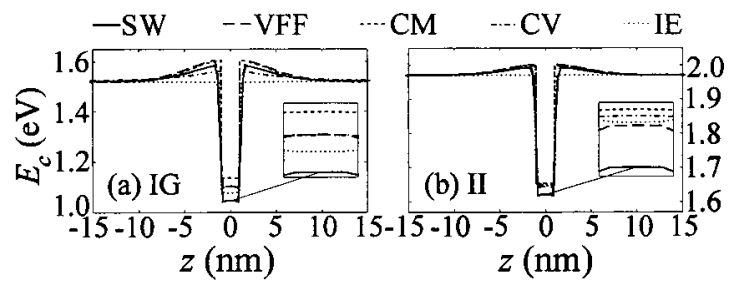

FIG. 9. Variation of the conduction band edge along the [001] direction in the InAs/GaAs and InP/InGaP QD's. (a) The bottom of the conduction band in InAs/GaAs QD. (b) The conduction band edge in the InP/InGaP QD. Different lines correspond to the different methods for the calculation of the strain, as indicated in the figure, and explained in the caption of Fig. 4.

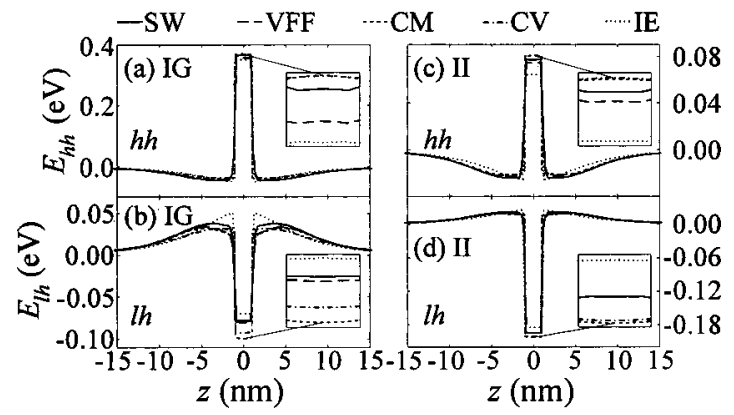

FIG. 10. The profiles of the two topmost valence bands along the [001] direction. Left-hand side panel: InAs/GaAs QD: (a) the heavy-hole band edge and (b) the light-hole band edge. Right-hand side panel: $\mathrm{InP} / \mathrm{InGaP}$ QD: (c) the variation of the top of the heavy-hole band and (d) the top of the light-hole band as it varies along the $z$ coordinate. The same line conventions as in Fig. 4 are used.

finite decay length in the matrix near the dot-matrix boundary. Our findings qualitatively agree with the results for the large pyramidal QD's. ${ }^{29,41}$

Along the $z$ axis, all shear strains are zero, $\varepsilon_{x x} \equiv \varepsilon_{y y}$, and, consequently, the valence band edges can be calculated by the IE theory for the [001] direction. The valence band edges extracted from the five models agree very well [see Figs. 10(a)-10(d)]. As for the conduction band, because all shear strains are zero, the displayed band edges correspond to the effective potentials. The heavy holes are confined in the barrier, while for the light holes, the potential wells are formed in the matrix near the disk boundary. The latter are very shallow and wide, hence the light holes may significantly contribute to the hole ground state for rather thin QD's, as supported by calculations of the electronic structure in the InP/InGaP QD's. ${ }^{34}$ All the potentials are nearly constant inside the dot, and the difference between the effective potentials for the heavy holes calculated by the different models is rather small in both dots. Related to the distribution of the strains along the $z$ direction shown in Fig. 6, the signs of the second derivative of the heavy and light holes edges calculated by all models change in the matrix, but this change is more pronounced in the case of CM and CV models than for the VFF and SW models, and only a slight variation of the curvature is noticed for the IE calculated strain distribution.

For the electron and the heavy-hole confinement the most relevant part is around the center of the QD. The normal strains at $\rho=0, z=0$ as they vary with the height of the QD in InAs/GaAs and InP/InGaP structures, are shown in Fig. 11. Due to the large computation time needed for the SW calculation, only the results extracted from other models are included in Fig. 11. Nonetheless, even those four calculations provide a lot of details, which are listed next. First, both $\varepsilon_{x x}(0,0)$ and $\varepsilon_{z z}(0,0)$ calculated by the IE theory have the largest slopes. This implies that the strongest dependence of the energy levels in the conduction band (depending on the hydrostatic strain) is expected to occur with variation of the QD height if the IE theory is employed for the calculation, as recently demonstrated. ${ }^{34}$ Second, the IE curves are linear, while rather small nonlinearities are produced by the other three models. The most pronounced nonlinearity is de- 


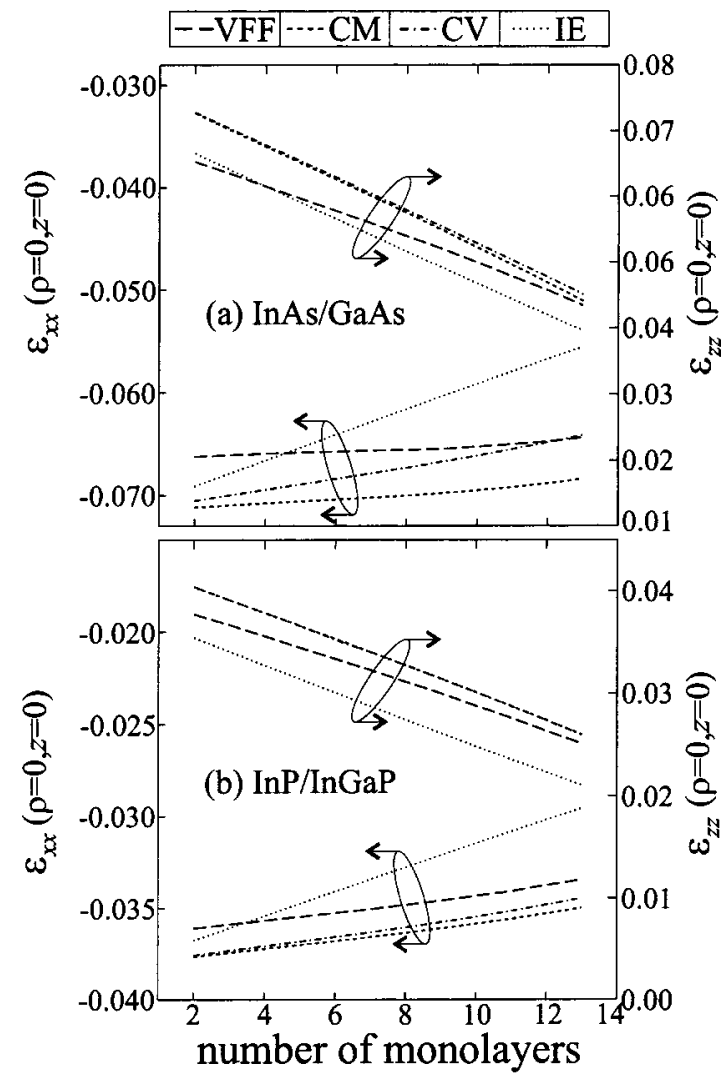

FIG. 11. The normal strains in the center of the QD calculated by the VFF, $\mathrm{CM}, \mathrm{CV}$, and IE models as they vary with the disk height. (a) The variation of the disk center strains in the InAs/GaAs QD. (b) The strains computed by the four models in the center of the InP/InGaP QD.

livered by the VFF-type calculation. Third, the VFF curve for $\varepsilon_{x x}(0,0)$ is parallel to the CM curves in both QD's, while $\varepsilon_{z z}(0,0)$ calculated by the CM model approaches the VFF result. Fourth, due to the smaller slopes of the VFF curves for $\varepsilon_{x x}(0,0)$, there are crossings between these curves and those produced by the IE model. $\varepsilon_{z z}(0,0)$ calculated by the VFF model crosses the IE curve for the InAs/GaAs QD, but no crossing occurs for the InP/InGaP QD's for 2-13 ML high QD's, and the VFF and CV curves for $\varepsilon_{x x}(0,0)$ in InAs/ GaAs QD cross at $\sim 12$ ML's. Fifth, the agreement between the CV and VFF calculated $\varepsilon_{x x}(0,0)$ and $\varepsilon_{z z}(0,0)$ is obviously better for the higher QD's. Sixth, the difference between the CM and CV calculated curves for $\varepsilon_{x x}(0,0)$ increases in both QD's when the QD height increases, but this increase is smaller for InP/InGaP QD's. Such behavior may be ascribed to the smaller misfit strain in the InP/InGaP QD's. Seventh, due to the same Poisson ratio in the CM and $\mathrm{CV}$ cases and rather small storage of energy by the shear, the $\varepsilon_{z z}(0,0)$ is larger if the $\mathrm{CV}$ model is used for the calculation, but the $\mathrm{CM}$ and $\mathrm{CV}$ calculated curves nearly coincide for the case of $\mathrm{InP} / \mathrm{InGaP}$.

In order to demonstrate the distribution of the strain in a vertical stack of QD's, Figs. 12(a) and 12(b) show the edges of the conduction and the valence bands along the $z$ direction in a system consisting of two $2 \mathrm{~nm}(\approx 7 \mathrm{ML})$ high InP QD's separated by a $2 \mathrm{~nm}$ thick layer of InGaP. The interaction of the strains around the two disks increases the energy of the

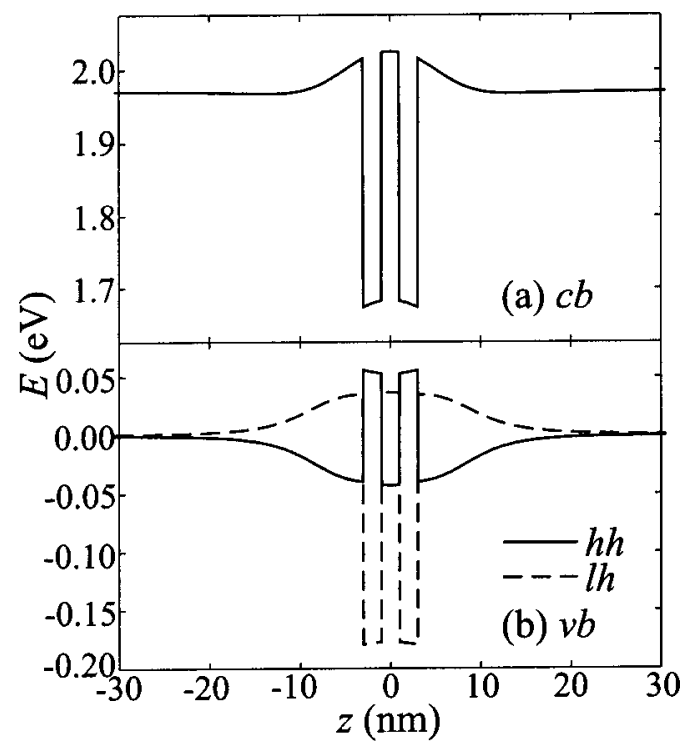

FIG. 12. The conduction and valence band edges along the $z$ direction in a stack consisting of two $2 \mathrm{~nm}$ high InP QD's and a $2 \mathrm{~nm}$ thick InGaP spacer between the dots. (a) The conduction band edge. (b) The edges of the heavy(solid curve) and the light-hole (dashed curve) bands.

bottom of the conduction band and decreases the energy of the top of the heavy-hole band. Therefore, the strain fields of the two QD's act jointly to diminish the quantum-mechanical coupling between electrons. The electrons and the heavy holes are located inside the disks, while the light holes are located in the matrix near the disk-matrix boundary, but the strain distribution also favors the confinement of the light holes in the spacer layer.

The strain field varies with the separation between the disks, as clearly indicated in Fig. 13, where the energies of the edge of the conduction band in the center of the disk and the center of the spacer layer are shown. These energies increase when the separation between the disks decreases. Therefore, if there is no quantum-mechanical coupling, the energies of the states in the conduction band would be

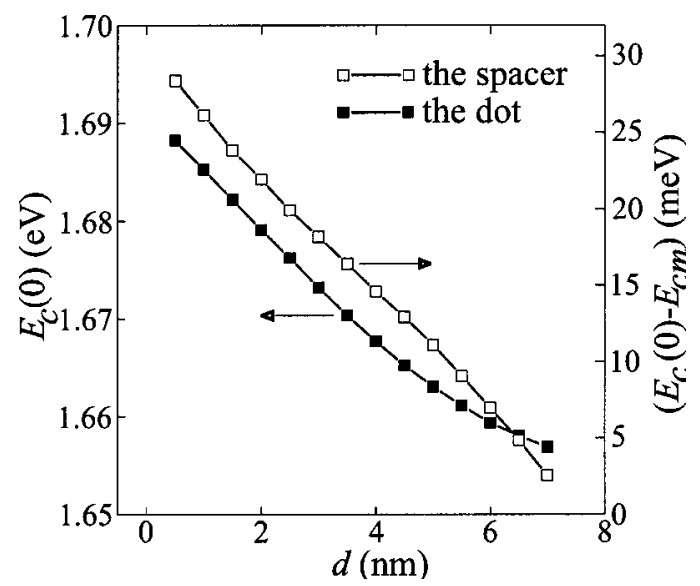

FIG. 13. The energies of the conduction band bottom in the center of the spacer layer (open squares) and the center of the QD (relative to the bottom of the conduction band in the matrix $E_{c m}$, solid squares) in a QD stack made up of two $2 \mathrm{~nm}$ high $\mathrm{InP} / \mathrm{InGaP}$ disks as they vary with the spacer thickness. 


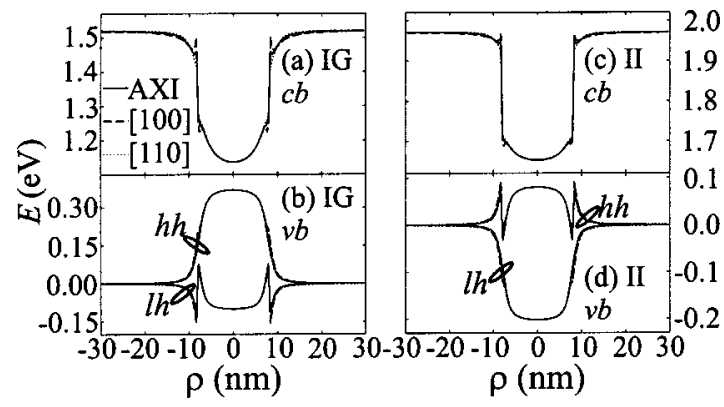

FIG. 14. The variation of the band edge energies in the InAs/GaAs and InP/InGaP QD's along the [100] and [110] directions, denoted, respectively, by the dashed and dotted lines, are compared with the result of the axial approximation, denoted by the solid line. Left-hand side panel: InAs/GaAs QD: (a) the bottom of the conduction band and (b) the edges of the valence bands. Right-hand side panel: InP/InGaP QD: (c) the edge of the conduction band and (d) the heavy-hole and light-hole band edges.

shifted upward. The downward shifts of the transition energies found experimentally ${ }^{12}$ indicate that the coupling or/and the Coulomb interaction between the electron and the hole is much stronger and suppresses the decrease of the confinement due to the strain.

As shown in Fig. 3, $\varepsilon_{\|}$and $\varepsilon_{z z}$ are almost axially symmetric, while $\varepsilon_{x y}$ exhibits a sharp drop at the lateral sides of the disk. Moreover, $\varepsilon_{x x}$ and $\varepsilon_{y y}$ are almost equal in both the disk and the matrix, while they differ near the lateral sides of the QD. Those findings led us to introduce the axial approximation of the kinetic part of the multiband Hamiltonian, which is enforced by replacing the $\varepsilon_{\|}$and $\varepsilon_{z z}$ by their angular averages given in Eq. (13), and neglecting the terms proportional to the off-diagonal strains. Such a procedure leads to the diagonal multiband Hamiltonian at $\mathbf{k}=0$ :

$$
H=\operatorname{diag}(P+Q, P-Q, P+Q, P-Q),
$$

where $P$ and $Q$ are given by:

$$
P=a_{v}\left(2\left\langle\varepsilon_{\|}\right\rangle+\left\langle\varepsilon_{z z}\right\rangle\right), \quad Q=b\left(\left\langle\varepsilon_{\|}\right\rangle-\left\langle\varepsilon_{z z}\right\rangle\right) .
$$

In order to demonstrate the usefulness of this approximation, in Figs. 14(a)-14(d) we compare the band edges for the [100] and [110] directions calculated by the CM model and extracted from Eqs. (12a) and (12b) (denoted by the dashed and dotted curves, respectively), with the band edges obtained by the axial approximation (denoted by the solid line) for the InAs/GaAs and InP/InGaP QD's. The results for the band edges produced by the axial approximation are obviously very close to the band edges for the [100] and [110] directions. Slight differences between the three curves exist near the radial periphery of the disk.

\section{SUMMARY AND CONCLUSION}

The strains calculated from the isotropic elasticity theory, the anisotropic CM model, the valence force field model and the atomistic model based on SW potentials in cylindrical QD's were compared. We found rather good agreement between the shapes of the strain tensor components calculated by the four methods, while quantitative discrepancies were found inside the disk and near the diskmatrix boundary. The strains in the disk center as calculated by the valence force field model exhibit crossings with the curves for these strains obtained by the isotropic elasticity theory. These crossings indicate that the mutual ordering of the electron and the hole energy levels calculated by these two methods may substantially change with the height of the quantum dot. The variation of the strains with the dot height has the largest slope if the IE theory is employed for the strain calculation, implying that the largest increase of the electron energies is expected, as recently found. ${ }^{34}$ Moreover, we found that the agreement between the different models is better for InP/InGaP QDs, which we ascribe to the smaller lattice mismatch in this system than in the InAs/GaAs QD's. Both $\varepsilon_{\|}$and $\varepsilon_{z z}$ are nearly axially symmetric, which leads to negligible deviations of the conduction and the valence band edges from axial symmetry. This leads to the important conclusion that for circularly symmetric self-assembled QD's the axial approximation of the multiband effective-mass Hamiltonian may be used.

In vertical stacks of QD's, we found that the strain field of the individual QD's tends to add up, resulting in an increase of the strain inside the dots. This lifts up the effective potential inside the QD's and between the dots. The net effect is that this counteracts the quantum-mechanical coupling between the dots.

We may conclude that for a better agreement with experiment, atomistic strain calculations are needed, but in InP/InGaP QD's, the small difference between the strains and band edges calculated by the different models may validate the usage of continuum models. Indeed, we recently found that the CM and IE theories reproduce the same features in the electron and hole spectra. ${ }^{34}$ The same shapes for the strain distribution delivered by the $\mathrm{CM}$ and atomistic models indicate that the same distributions for the electron and hole densities in single and coupled QD's will be obtained if the atomistic models are employed for that purpose.

\section{ACKNOWLEDGMENTS}

This work was partially supported by the Flemish Science Foundation (FWO-V1), the Belgian Interuniversity Attraction Poles (IUAP), the University of Antwerp (GOA and VIS), and the European Commission GROWTH program NANOMAT project, Contract No. G5RD-CT-2001-00545. One of the authors (M. T.) was supported by a postdoctoral fellowship of the University of Antwerp (BOF/UIA) and another (K. L. J.) by the "Instituut voor de aanmoediging van Innovatie door Wetenschap en Technologie in Vlaanderen" (IWT-V1). Discussions with Professor J. H. Davies, Professor Z. Ikonić, Professor M. Grundmann, A. Schliwa, Dr. M. Hayne, M. Migliorato, and Professor V. Moshchalkov are gratefully acknowledged.

\footnotetext{
${ }^{1}$ L. Jacak, P. Hawrylak, and A. Wójs, Quantum Dots (Springer, Berlin, 1998).

${ }^{2}$ D. Bimberg, M. Grundmann, and N. N. Ledentsov, Quantum Dot Heterostructures (Wiley, London, 1999).

${ }^{3}$ T. Riedl, E. Fehrenbacher, A. Hangleiter, M. K. Zundel, and K. Eberl, Appl. Phys. Lett. 73, 3730 (1998).

${ }^{4}$ R. L. Sellin, C. Ribbat, M. Grundmann, N. N. Ledentsov, and D. Bimberg, Appl. Phys. Lett. 78, 1207 (2001).
} 
${ }^{5}$ H. C. Liu, M. Gao, J. McCaffrey, Z. R. Wasilewski, and S. Fafard, Appl. Phys. Lett. 78, 79 (2001).

${ }^{6}$ M. Borgstrom, T. Bryllert, T. Sass, B. Gustafson, L.-E. Wernersson, W. Seifert, and L. Samuelson, Appl. Phys. Lett. 78, 3232 (2001).

${ }^{7}$ M. Grundmann, O. Stier, and D. Bimberg, Phys. Rev. B 52, 11969 (1995).

${ }^{8}$ Q. Xie, A. Madhukar, P. Chen, and N. P. Kobayashi, Phys. Rev. Lett. 75, 2542 (1995).

${ }^{9}$ G. S. Solomon, J. A. Trezza, A. F. Marshall, and J. S. Harris, Phys. Rev. Lett. 76, 952 (1996).

${ }^{10}$ N. N. Ledentsov et al., Phys. Rev. B 54, 8743 (1996).

${ }^{11}$ R. Heitz, A. Kalburge, Q. Xie, M. Grundmann, P. Chen, A. Hoffmann, A. Madhukar, and D. Bimberg, Phys. Rev. B 57, 9050 (1998).

${ }^{12}$ M. Hayne, R. Provoost, M. K. Zundel, Y. M. Manz, K. Eberl, and V. V. Moshchalkov, Phys. Rev. B 62, 10324 (2000).

${ }^{13}$ J. G. Belk, J. L. Sudijono, X. M. Zhang, J. H Neave, T. S. Jones, and B. A. Joyce, Phys. Rev. Lett. 78, 475 (1997).

${ }^{14}$ H. Yamaguchi, J. G. Belk, X. M. Zhang, L. J. Sudijono, M. R. Fahy, T. S. Jones, D. W. Pashley, and B. A. Joyce, Phys. Rev. B 55, 1337 (1997).

${ }^{15}$ P. B. Joyce, T. J. Krzyzewski, G. R. Bell, B. A. Joyce, and T. S. Jones, Phys. Rev. B 58, R15981 (1998).

${ }^{16}$ B. A. Joyce, D. D. Vvedensky, T. S. Jones, M. Itoh, G. R. Bell, and J. G. Belk, J. Cryst. Growth 201, 106 (1999).

${ }^{17}$ K. Georgsson, N. Carlsson, L. Samuelson, W. Seifert, and L. R. Wallenberg, Appl. Phys. Lett. 67, 2981 (1995).

${ }^{18}$ M. K. Zundel, P. Specht, K. Eberl, N. Y. Jin-Phillipp, and F. Phillipp, Appl. Phys. Lett. 71, 2972 (1997).

${ }^{19}$ F. Hatami, M. Grundmann, N. N. Ledentsov, F. Heinrichsdorff, R. Heitz, J. Böhrer, D. Bimberg, S. S. Ruvimov, P. Werner, V. M. Ustinov, P. S. Kop'ev, and Z. I. Alferov, Phys. Rev. B 57, 4635 (1998).

${ }^{20}$ S. M. North, P. R. Bridon, M. A. Cusack, and M. Jaros, Phys. Rev. B 58, 12601 (1998).

${ }^{21}$ O. G. Schmidt and K. Eberl, Phys. Rev. B 61, 13721 (2000).

${ }^{22}$ J. P. McCaffrey, M. D. Robertson, P. J. Poole, B. J. Riel, and S. Fafard, J. Appl. Phys. 90, 1784 (2001).

${ }^{23}$ B. Jogai, J. Appl. Phys. 88, 5050 (2000).

${ }^{24}$ B. Jogai, J. Appl. Phys. 90, 699 (2001).

${ }^{25}$ F. Glas, J. Appl. Phys. 90, 3232 (2001).

${ }^{26}$ J. H. Davies, D. M. Bruls, J. W. A. M. Vugs, and P. M. Koenraad, J. Appl. Phys. 91, 4171 (2002).

${ }^{27}$ D. M. Bruls, P. M. Koenraad, M. Hopkinson, J. H. Wolter, and H. W. M. Salemink, Appl. Surf. Sci. 190, 258 (2002).

${ }^{28} \mathrm{H}$. Jiang and J. Singh, IEEE J. Quantum Electron. 34, 1188 (1998).

${ }^{29}$ C. Pryor, Phys. Rev. B 57, 7190 (1998).

${ }^{30}$ C. Pryor, M.-E. Pistol, and L. Samuelson, Phys. Rev. B 56, 10404 (1997).

${ }^{31}$ A. Wojs, P. Hawrylak, S. Fafard, and L. Jacak, Phys. Rev. B 54, 5604 (1996).

${ }^{32}$ M. Bayer, P. Hawrylak, K. Hinzer, S. Fafard, M. Korkusinski, Z. R. Wasilewski, O. Stern, and A. Forchel, Science 291, 451 (2001).

${ }^{33}$ M. Korkusiński and P. Hawrylak, Phys. Rev. B 63, 195311 (2001).

${ }^{34}$ M. Tadić, F. M. Peeters, and K. L. Janssens, Phys. Rev. B 65, 165333 (2002).

${ }^{35}$ A. D. Andreev, J. R. Downes, D. A. Faux, and E. P. O. Reilly, J. Appl. Phys. 86, 297 (1999).
${ }^{36}$ P. N. Keating, Phys. Rev. 145, 637 (1966).

${ }^{37}$ L. D. Landau and E. M. Lifshitz, Theory of Elasticity (ButterworthHeinemann, Oxford, 1995).

${ }^{38}$ G. S. Pearson and D. A. Faux, J. Appl. Phys. 88, 730 (2000).

${ }^{39}$ J. R. Downes, D. A. Faux, and E. P. O'Reilly, J. Appl. Phys. 81, 6700 (1997).

${ }^{40}$ J. H. Davies, J. Appl. Phys. 84, 1358 (1998).

${ }^{41}$ C. Pryor, J. Kim, L. W. Wang, A. J. Williamson, and A. Zunger, J. Appl. Phys. 83, 2548 (1998).

${ }^{42}$ R. M. Martin, Phys. Rev. B 1, 4005 (1970).

${ }^{43}$ F. H. Stillinger and T. A. Weber, Phys. Rev. B 31, 5262 (1985).

${ }^{44}$ J. Tersoff, Phys. Rev. Lett. 56, 632 (1986).

${ }^{45}$ J. Tersoff, Phys. Rev. B 39, 5566 (1989).

${ }^{46}$ Y. Kikuchi, H. Sugii, and K. Shintani, J. Appl. Phys. 89, 1191 (2001).

${ }^{47}$ L. A. Zepeda-Ruiz, D. Maroudas, and W. H. Weinberg, J. Appl. Phys. 85, 3677 (1999)

${ }^{48}$ L. A. Zepeda-Ruiz, R. I. Pelzel, B. Z. Nosho, W. H. Weinberg, and D. Maroudas, J. Appl. Phys. 90, 2689 (2001).

${ }^{49}$ W. Yu and A. Madhukar, Phys. Rev. Lett. 79, 905 (1997); ibid. 79, 4939 (1997).

${ }^{50}$ M. A. Migliorato, A. G. Cullis, M. Fearn, and J. H. Jefferson, Phys. Rev. B 65, 115316 (2002).

${ }^{51}$ J. Tersoff, Phys. Rev. Lett. 79, 4934 (1997).

${ }^{52}$ X. Su, R. K. Kalia, A. Nakano, P. Vashishta, and A. Madhukar, Appl. Phys. Lett. 78, 3717 (2001).

${ }^{53}$ X. Su, R. K. Kalia, A. Nakano, P. Vashishta, and A. Madhukar, Appl. Phys. Lett. 79, 4577 (2001).

${ }^{54}$ L. W. Wang, A. J. Williamson, A. Zunger, H. Jiang, and J. Singh, Appl. Phys. Lett. 76, 339 (2000).

${ }^{55}$ T. Saito, J. N. Schulman, and Y. Arakawa, Phys. Rev. B 57, 13016 (1998).

${ }^{56}$ S. J. Sun and Y. -C. Chang, Phys. Rev. B 62, 13631 (2000).

${ }^{57}$ O. G. Schmidt, K. Eberl, and Y. Rau, Phys. Rev. B 62, 16715 (2000).

${ }^{58}$ S. Christiansen, M. Albrecht, H. P. Strunk, and H. J. Maier, Appl. Phys. Lett. 64, 3617 (1994).

${ }^{59}$ T. Benabbas, Y. Androussi, and A. Lefebvre, J. Appl. Phys. 86, 1945 (1999).

${ }^{60}$ R. Virkkala, K. Maijala, and J. Tulkki, Phys. Rev. B 62, 6932 (2000).

${ }^{61}$ A. E. Romanov, G. E. Beltz, W. T. Fischer, P. M. Petroff, and J. S. Speck, J. Appl. Phys. 89, 4523 (2001).

${ }^{62}$ I. P. Ipatova, V. G. Malyshkin, and V. A. Shchukin, J. Appl. Phys. 74, 7198 (1993).

${ }^{63}$ D. Sander, Rep. Prog. Phys. 62, 809 (1999).

${ }^{64}$ K. L. Janssens, B. Partoens, and F. M. Peeters, Phys. Rev. B 64, 155324 (2001).

${ }^{65}$ M. Ichimura, Phys. Status Solidi A 153, 431 (1996).

${ }^{66}$ M. Karimi, T. Kaplan, M. Mostoller, and D. E. Jesson, Phys. Rev. B 47, 9931 (1993).

${ }^{67}$ C. Y.-P. Chao and S. L. Chuang, Phys. Rev. B 46, 4110 (1992).

${ }^{68}$ Y. Zhang, Phys. Rev. B 49, 14352 (1994).

${ }^{69}$ D. A. Broido and L. J. Sham, Phys. Rev. B 31, 888 (1985).

${ }^{70}$ Z. Ikonić, V. Milanović, and D. Tjapkin, Phys. Rev. B 46, 4285 (1992).

${ }^{71}$ J. Groenen, C. Priester, and R. Carles, Phys. Rev. B 60, 16013 (1999). 